\title{
In-Situ Metallography and Replication of Microstructures For Condition Assessment \& Remaining Life Analysis.
}

\author{
*Yashwant Ranaware, **Girish Shejale \\ *Manager-Life Extension Services, Sagar Complex, Kasarwadi, Thermax Babcock \& Wilcox \\ Limited, India - 411034. \\ **Assistant Manager-Life Extension Services, Sagar Complex, Kasarwadi, Thermax Babcock \& \\ Wilcox Limited, India- 411034.
}

\section{Introduction:}

As a plant ages, high temperature headers steam piping and other critical components must be periodically evaluation for failure initiation. Proper evaluation of component condition can permit planning for replacement in-kind, maintenance scheduling or material upgrading. Insitu Metallography / Replication and evaluation is introduced as one of the core technical requirements in the life assessment programme of power and process boilers [a]. A replica is essentially a "fingerprint" of the surface under examination and can be used to detect cracking, creep cavitation, porosity, inclusions and other similar defects that are undetectable by other non destructive techniques. Replication thus provides an early warning of impending failure and identifies the active mechanism of failure. Often final mechanical polishing closes many creep cavities or fine cracks due to a thin plastically deformed layer inherent in the very polishing process in the field conditions . Hence serious errors by under estimating the actual damage will result . Electropolishing (EP) can overcome this problem. Versatile All Position IN-situ Electropolisher (VAPINE), developed recently has several new features for obtaining excellent quality metallographic surface. VAPINE has been used effectively and successfully in several plant sites and also for laboratory samples of standard sizes.

\section{Experimental:}

The high quality final polishing is done by electrolytic polishing by Versatile All Position IN-situ Electro-Polisher ['VAPINE"] which consists of precision machined austenitic stainless steel A 316 components as illustrated in Fig. 1. The stainless steel main body (1) has a precision screwed teflon insert (9) at the bottom for letting out the electrolyte (8) through mutually perpendicular four holes (11). The size of the Orifice (10) can be changed easily to control the flow to desired level e.g.,. $2 \mathrm{ml}$ - $5 \mathrm{ml} / \mathrm{min}$. by changing the insert (9) with different bore diameter. The top lid (2) is fitted to main body by a pair of screws (7) and a Titanium handle (16) is fitted using bolt and nut (6) at the point (3) enabling electrolyte inlet flow (5) easily by accomplishing air escape through air vent plastic tube (4) fitted to the diametrically opposite point. The titanium handle has an insulation (17) at the handling zone and the positive lead of $+42 \mathrm{~V} \mathrm{D}$. C power source is connected (19) at the time of electro-polishing. The teflon shroud (14) will enable to do overhead electro-polishing without spills and at that time the top port holes 5 and 4 are disabled by closing with screws. The electrolyte sucked by tampon (12) and retained by grooves at the bottom of body (1) is sufficient for overhead electro-polishing. The spilled electrolyte is collected by the shroud (14) and this is not reused. For normal, flat and inclined position electro-polishing the shroud (14) is not helpful and hence can be removed. The meniscus of electrolyte (13) after flow via tampon (12) is maintained by keeping a gap of 0.5 to $2.0 \mathrm{~mm}$ between cathode body (1) and the component surface (15) with 600 grit $\mathrm{SiC}$ paper surface finish. The anodic connection (18) is given to the component by a magnetic holder for 
low alloy steels or by keeping a weight on the anode metal strip for non-magnetic materials. Alternatively, a suitable conducting clamp can be used for the letter.

The entire assembly of VAPINE is dipped in a ice-chilled electrolyte of 150-200 ml volume, kept in a stainless steel tumbler surrounded ice in a compact thermal jug. The titanium handle (16) is moved up and down for a few seconds such that electrolyte flows through inlet (5) and gets collected at core (8) with a capacity of about $15 \mathrm{ml}$. The VAPINE unit is taken out keeping electrode connections as in Fig. 1 and the tampon (12) is allowed to come close to the component surface (15) by keeping a meniscus (13) at a distance of 0.5 to $2 \mathrm{~mm}$. The component's surface is chilled by ice and wiped dry VAPINE is slowly moved up and down, and also in a circular fashion covering an area of about 15 $\mathrm{mm}$ diameter circle. It will take a few minutes to discharge electrolyte during electro-polishing. The anodic dissolution and polishing occurs at the rate of 0.8 to $1 \mu \mathrm{m}$ per minute in a controlled fashion. The clear electrolyte will become golden yellow after anodic reaction at the meniscus point for most of steels. VAPINE is compact and light with weight device weighing less than 500 gram, and hence very convenient for electro-polishing at many with limited space. Approximately 10 minutes electropolishing is adequate to remove all 600 grit $\mathrm{SiC}$ scratches and to get brilliantly polished spot of about $15 \mathrm{~mm}$ diameter. After etching, the component is examined directly by a portable microscope for observing the microstructures upto $400 \mathrm{X}$ magnifications. However, it is beneficial and preferable to take a replica using a transparent acetate tape of $25 \mathrm{~mm}$ width and thickness of $0.1 \mathrm{~mm}$ or $0.03 \mathrm{~mm}$ [b].

\section{Conclusion:}

Thus, it can be concluded that high quality in-situ metallography is achievable using VAPINE, leading to plastic deformation free surface, which yielded high resolution microstructures after examination of several alloys of direct metal and gold sputtered replica using light metallurgical microscope and SEM. In-situ metallography significantly helps to maintain quality standards of high temperature pressure parts of low alloy steels and other alloys of fossil power and petrochemical plants by recommending the strategy - Run or Repair or Replace [c].

\section{References:}

o [a] Viswanathan R, Damage Mechanisms and Life Assessment of High Temperature Components, ASM International, Ohio (1989) pp.182-263

o [b] Venkataraman G, Practical Metallography (Germany), Part-I, Vol.18 (1981) pp.237-245, PartII pp.342-353, Part III,pp.434-442.

o [c] Venkataraman G, Creep Damage Evaluation and Condition Assessment of Fossil Power and Process Plant Components by In-situ Metallography.

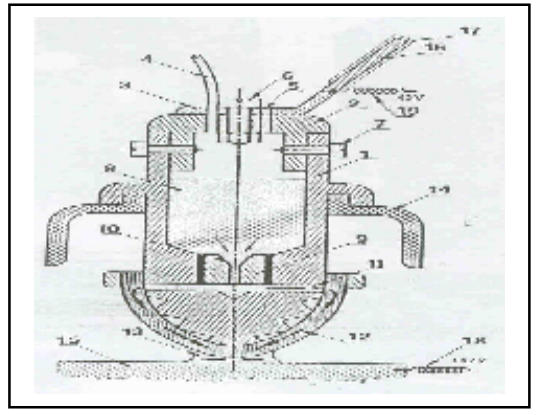

Figure 1: Schematic Section illustrating electropolishing principle of VAPINE (Versatile All In-Situ Electropolisher) 